\title{
Identifying a spin liquid on the Kagome lattice using quantum entanglement Featured papers:
}

1. Identifying Topological Order by Entanglement Entropy, Hong-Chen Jiang, Zhenghan Wang, and Leon Balents; arXiv:1205.4289

2. Nature of the Spin Liquid Ground State of the $S=1 / 2$ Kagome Heisenberg Model Stefan Depenbrock, Ian P. McCulloch, and Ulrich Schollwöck, arXiv:1205.4858.

\section{Recommended with a commentary by Ashvin Vishwanath, UC Berkeley}

Recent years have seen a growing synergy between quantum information and condensed matter physics. Ideas from quantum information have helped deepen understanding of the DMRG (density matrix renormalization group) method and have provided new diagnostics for phases of matter with no Landau order parameter. The featured papers discuss an interesting combination of these two themes that is employed to identify the ground state of the Kagome antiferromagnet as a topological spin liquid.

The problem of the Kagome quantum antiferromagnet is easily stated, but has frustrated researchers for decades. What is the ground state of antiferromagnetically coupled $\mathrm{S}=1 / 2$ moments on the Kagome lattice (Fig 1A)?

$$
H=J \sum_{\langle i j\rangle} \mathbf{S}_{i} \cdot \mathbf{S}_{j}
$$

Various outcomes ranging from magnetic order to valence bond solids and quantum spin liquids of different types have been proposed. Recently, additional impetus was provided by the discovery of materials which at first cut may be modeled by Eqn (1). In a recent numerical breakthrough made possible by advances in the DMRG technique, Yan,Huse and White [1] showed that the ground state is gapped and shows no sign of valence bond or magnetic order at the largest system sizes. A natural conclusion is that the ground state is the long sought after quantum spin liquid, an exotic states of matter that differs at a fundamental topological level from conventional phases. The simplest possibility that preserves all symmetries is the $Z_{2}$ spin liquid, whose basic properties are described by an emergent $Z_{2}$ gauge field. This is an Ising version of electrodynamics, where electric fields take values 0,1 and magnetic flux is quantized to $0, \pi$. Pictorially - an electric field is 1 or 0 


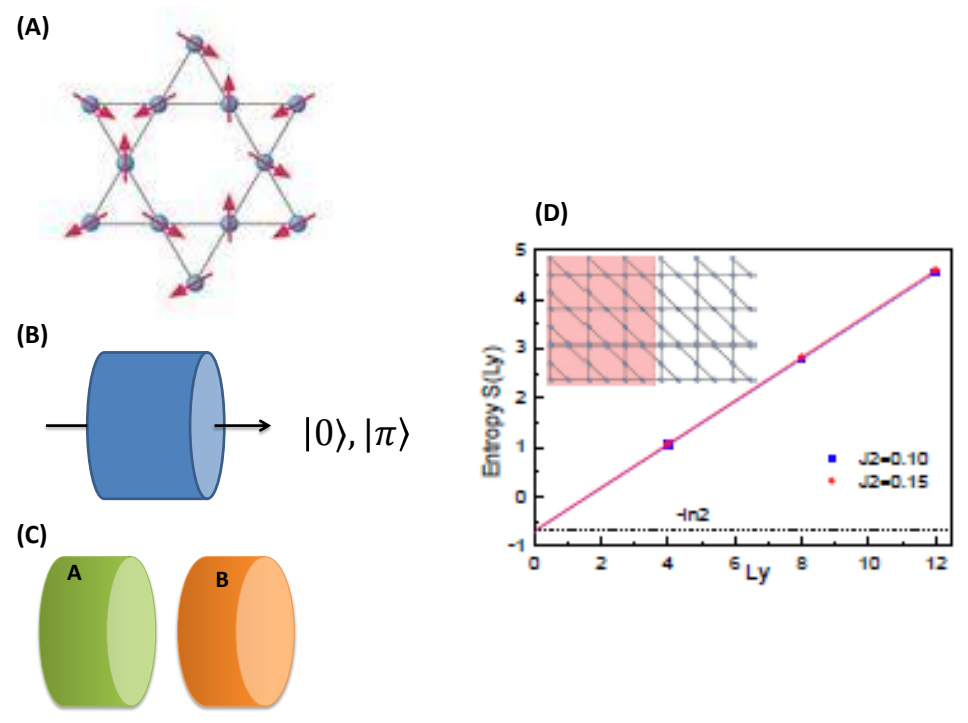

FIG. 1: (A) The Kagome antiferromagnet (B) the two ground states of a $\mathrm{Z}_{2}$ spin liquid defined on a cylindrical system, with emergent Ising flux $0, \pi$ threading the cylinder.(C) an entanglement cut that divides the cylinder in two, used in the featured references. (D) numerical data (from featured Ref 1.) of the entanglement entropy for such a cut plotted against circumference of the cylinder in units of lattice spacing. The intercept is the topological entanglement entropy.

on bond depending on whether the spins at the ends form singlets or not. Electric charges are unpaired spins or spinons. However, identifying a positive signature of a quantum spin liquid was left open. Furthermore a puzzling aspect was that an expected ground state degeneracy on the cylinder was not seen. This degeneracy may be pictured as a solenoid of 0 or $\pi$ Ising flux inserted in the cylinder (Fig 1B).

The highlighted references provide strong positive evidence of a quantum spin liquid on the Kagome lattice by computing the topological entanglement entropy (TEE) and showing it is consistent with what is expected for a topological spin liquid of the $\mathrm{Z}_{2}$ type. The entanglement entropy measure how strongly entangled a subregions $A$ is with the rest of the system. In general for a gapped phase at zero temperature, this scales with the length of the boundary $L_{A}$ of $A$. For a smooth boundary, the topological entanglement entropy appears as a subleading constant, that does not scale with the length of the boundary.

$$
S_{A}=\alpha L_{A}-\gamma
$$

A non-zero topological entanglement entropy only appears for topologically ordered states. 
These are gapped phases which also display ground state degeneracy when defined on closed surfaces. The Laughlin fractional quantum Hall states are topologically ordered, i.e. have $\gamma>0$ but integer Quantum Hall phases, topological insulators and regular band insulators are not and have $\gamma=0$.

For a $Z_{2}$ topological phase one expects $\gamma=\log 2$. Intuitively this may be understood as follows. The entanglement entropy measures our lack of knowledge due to removing a subregion. In a gauge theory, one has the Gauss law constraint, and in the ground state where no charges are present $\nabla \cdot E=0$. In the Ising gauge theory this equation is understood as equality mod 2 . Thus one has more information than one might naively think because any field configuration must obey the constraint. The entanglement entropy is therefore reduced by this one bit of information, hence $\gamma=\log 2$. As a practical matter though, on a lattice, a typical boundary will not be smooth and contain corners. Then, one cannot directly use the above definition, and a more complicated subtraction scheme [2] is needed which is impractical for DMRG. However, there is one smooth boundary cut possible in a lattice system. If the system is, say, defined on a cylinder (i.e. with periodic boundary conditions along one direction) which is then divided into two cylindrical regions (Fig 1C). Then, finite size scaling of entanglement entropy with the cylinder circumference could potentially be used to extract $\gamma$. Indeed, this is the method adopted by the references above in the context of the Kagome antiferromagnet. The value of $\gamma$ that is obtained is very close to $\log 2$, in fact Ref 1 finds it to be within a percent of the expected value of 0.693.(Fig 1D)

While at first sight this appears to be in perfect agreement with theory, indicating a $\mathrm{Z}_{2}$ topological phase, the cylindrical geometry of subsystem $A$ introduces an extra complication. One can pick any linear combination of the doubly degenerate ground states and the topological entanglement entropy can range from 0 to $\log 2$ depending on the ground state[3]. The states for which $\gamma=\log 2$ are the ones with definite flux inside the cylinder (fig 1). these are termed Minimum entropy states (MES) since they have the maximum topological entanglement entropy, which lowers the total entropy. Any linear combination of the MES leads to a loss of information, and a corresponding increase in entropy. In a finite size system, there is a small splitting between the pair of ground states. Ref 1 argues that DMRG's well known preference for states with lower entanglement means it would lock into a single MES, even if it is slightly higher in energy than a linear combination. This is demonstrated by numerically simulating exactly soluble models like the Kitaev model. 
This could also explain the puzzling fact that the two ground states of the $\mathrm{Z}_{2}$ spin liquid are not observed in DMRG - the algorithm locks into one of the MES, and is prevented from exploring the other one by an entanglement barrier. Other topological phases, such as bosons in a flat Chern band at half filling where a bosonic Laughlin state is expected, were found by the same method to have the expected $\gamma=\frac{1}{2} \log 2$.

For the Kagome, the close match with the expected value of TEE implies a positive indication of a gapped spin liquid - very likely with $\mathrm{Z}_{2}$ topological order. (Ref 1 studies the Kagome slightly perturbed away from the nearest neighbor model, where it is believed to be in the same phase, while Ref 2 looked at the nearest neighbor antiferromagnet itself, with an improved DMRG algorithm that implements SU(2) symmetry). A puzzle that remains is why experimental realizations of the Kagome lattice typically appear quite different, eg without a spin gap. Nevertheless, the tools to simulate and diagnose exotic topologically ordered phases are rapidly progressing to the point where they may guide the search for such state in materials.

[1] S. Yan, D. Huse, S. White, Science 332, 1173 (2011).

[2] A. Kitaev and J. Preskill, Phys. Rev. Lett. 96, 110404 (2006). M. Levin, X.-G. Wen, Phys. Rev. Lett. 96, 110405 (2006).

[3] S. Dong, et al. JHEP 0805, 016 (2008).; Y. Zhang et al. Phys. Rev. B 85, 235151 (2012). (arXiv:11112342). 